PHYSICAL REVIEW D 85, 119901(E) (2012)

\title{
Erratum: Double charmonium production in exclusive bottomonia decays [Phys. Rev. D 80, 094008 (2009)]
}

\author{
V. V. Braguta, A. K. Likhoded, and A. V. Luchinsky \\ (Received 11 May 2012; published 4 June 2012)
}

DOI: 10.1103/PhysRevD.85.119901

PACS numbers: 12.38.- t, 12.38.Bx, 13.25.Gv, 99.10.Cd

In paper [1], we calculated the widths of the leading twist bottomonia decays to double charmonia in the framework of light cone expansion formalism (LC) and nonrelativictic QCD (NRQCD). We made the following errors in the calculation: (1) The factor $1 / 2$ was lost in the expressions for all amplitudes and the factor $1 / 4$ was lost in the expressions for all widths of double charmonium production in exclusive bottomonia decays. To correct this error one should divide all amplitudes by 2 and all widths by 4 . (2) The factor $\beta$ that accounts the phase space integration

$$
\beta=\frac{2\left|\vec{p}_{f}\right|}{M_{b b}}=\frac{\sqrt{M_{b b}^{2}-\left(m_{1}+m_{2}\right)^{2}} \sqrt{M_{b b}^{2}-\left(m_{1}-m_{2}\right)^{2}}}{M_{b b}^{2}},
$$

was lost in the expressions for all decay widths. In the last formula we used the designations $\vec{p}_{f}$ is the momentum of one of the final meson, and $M_{b b}$ and $m_{1,2}$ are masses of the bottomonium and final chamonia mesons, respectively. So, to correct this error all widths of the decays considered in [1] must be multiplied by the factor $\beta$.Within LC the hard scale $M$ of

TABLE I. The widths and branching fractions of the exclusive bottomonia decays into pair of charmonium mesons. In the second column the NRQCD predictions are presented. In the third and fourth columns the widths and branching fractions of the exclusive bottomonia decays in LC formalism are shown. The last column contains the branching fractions of inclusive $J / \psi$-pair production through intermediate charmonium states. The symbol "_-" in this column means that this decay is forbidden (for example, decay $\eta_{b} \rightarrow \eta_{c} \chi_{c 0} \rightarrow J / \psi J / \psi+X$ is absent since $\eta_{c}$ meson cannot decay into $J / \psi$ ) or its branching fraction is unknown (for example, in the case $\left.\eta_{b} \rightarrow h_{c} J / \psi\right)$.

\begin{tabular}{|c|c|c|c|c|}
\hline Reaction & $\Gamma_{\mathrm{NRQCD}}, \mathrm{eV}$ & $\Gamma_{\mathrm{LC}}, \mathrm{eV}$ & $\mathrm{Br}_{\mathrm{LC}}, 10^{-5}$ & $\mathrm{Br}_{\mathrm{LC}}(\psi \psi), 10^{-5}$ \\
\hline$\eta_{b} \rightarrow h_{c} \psi$ & $16 ._{-1.5}^{+2.3} \pm 8.4 \pm 8.1$ & 32. $\pm 2.6 \pm 6.1 \pm 8.2$ & 0.33 & - \\
\hline$\eta_{b} \rightarrow h_{c} \psi(2 S)$ & $7.8_{-0.72}^{+1.1} \pm 6.5 \pm 3.9$ & 16. $\pm 1.4 \pm 3.1 \pm 4.2$ & 0.17 & - \\
\hline$\eta_{b} \rightarrow \eta_{c} \chi_{c 0}$ & $13 ._{-2.7}^{+3.5} \pm 6.8 \pm 6.5$ & $9.1 \pm 0.73 \pm 4.6 \pm 2.3$ & 0.092 & - \\
\hline$\eta_{b} \rightarrow \eta_{c}(2 S) \chi_{c 0}$ & $6.3_{-1.3}^{+1.7} \pm 5.2 \pm 3.1$ & $4.3 \pm 0.36 \pm 3 . \pm 1.1$ & 0.043 & - \\
\hline$\eta_{b} \rightarrow \eta_{c} \chi_{c 2}$ & $3.6_{-1.1}^{+1.1} \pm 8.4 \pm 1.8$ & 18. $\pm 1.4 \pm 8.7 \pm 4.5$ & 0.18 & - \\
\hline$\eta_{b} \rightarrow \eta_{c}(2 S) \chi_{c 2}$ & $1.7_{-0.53}^{+0.54} \pm 4.1 \pm 0.86$ & $8.2 \pm 0.7 \pm 5.6 \pm 2.1$ & 0.083 & - \\
\hline$\eta_{b} \rightarrow \chi_{c 0} \chi_{c 1}$ & $2.3_{-0.29}^{+0.21} \pm 2.2 \pm 1.2$ & $4.4 \pm 0.38 \pm 2.3 \pm 1.1$ & 0.045 & $2.1 \times 10^{-4}$ \\
\hline$\eta_{b} \rightarrow \chi_{c 1} \chi_{c 2}$ & $0.93_{-0.21}^{+0.22} \pm 2.9 \pm 0.46$ & $8.6 \pm 0.73 \pm 4.3 \pm 2.2$ & 0.087 & 0.0062 \\
\hline$\chi_{b 0} \rightarrow \eta_{c} \chi_{c 1}$ & $1.9_{-0.27}^{+0.23} \pm 1.9 \pm 0.93$ & $9.8 \pm 0.25 \pm 4.8 \pm 2.5$ & 1.2 & - \\
\hline$\chi_{b 0} \rightarrow \eta_{c}(2 S) \chi_{c 1}$ & $0.9_{-0.13}^{+0.11} \pm 1.1 \pm 0.45$ & $5.9 \pm 1 . \pm 4 . \pm 1.5$ & 0.73 & - \\
\hline$\chi_{b 0} \rightarrow \chi_{c 0} \chi_{c 2}$ & $0.00015_{-0.00014}^{+0.0007} \pm 0.038 \pm 7.6 \times 10^{-5}$ & $0.14 \pm 0.034 \pm 0.07 \pm 0.034$ & 0.017 & $4.5 \times 10^{-5}$ \\
\hline$\chi_{b 0} \rightarrow \eta_{c} \eta_{c}$ & $7.9_{-0.57}^{+0.69} \pm 5.6 \pm 4$ & 10. $\pm 0.45 \pm 4.9 \pm 2.5$ & 1.3 & - \\
\hline$\chi_{b 0} \rightarrow \eta_{c} \eta_{c}(2 S)$ & $7.8_{-0.56}^{+0.68} \pm 7.5 \pm 3.9$ & 12. $\pm 2.1 \pm 8.3 \pm 3$ & 1.5 & - \\
\hline$\chi_{b 0} \rightarrow \eta_{c}(2 S) \eta_{c}(2 S)$ & $1.9_{-0.14}^{+0.16} \pm 2.8 \pm 0.94$ & $3.6 \pm 1.4 \pm 3 . \pm 0.91$ & 0.45 & - \\
\hline$\chi_{b 0} \rightarrow \psi \psi$ & $4.3_{-0.25}^{+0.28} \pm 5.7 \pm 2.2$ & 15. $\pm 0.68 \pm 0.51 \pm 3.8$ & 1.9 & 1.9 \\
\hline$\chi_{b 0} \rightarrow \psi \psi(2 S)$ & $4.3_{-0.25}^{+0.28} \pm 6.3 \pm 2.1$ & $20 . \pm 3.5 \pm 0.62 \pm 5$ & 2.5 & 1.4 \\
\hline$\chi_{b 0} \rightarrow \psi(2 S) \psi(2 S)$ & $1 ._{-0.06}^{+0.068} \pm 1.9 \pm 0.52$ & $6.5 \pm 2.5 \pm 0.18 \pm 1.6$ & 0.81 & 0.27 \\
\hline$\chi_{b 0} \rightarrow h_{c} h_{c}$ & $0.014_{-0.0035}^{+0.0025} \pm 0.021 \pm 0.0071$ & $0.3 \pm 0.074 \pm 0.079 \pm 0.075$ & 0.037 & - \\
\hline$\chi_{b 0} \rightarrow \chi_{c 0} \chi_{c 0}$ & $0.006_{-0.0041}^{+0.0076} \pm 0.022 \pm 0.003$ & $0.035 \pm 0.0087 \pm 0.018 \pm 0.0088$ & 0.0044 & $7.4 \times 10^{-7}$ \\
\hline$\chi_{b 0} \rightarrow \chi_{c 1} \chi_{c 1}$ & $0.087_{-0.025}^{+0.037} \pm 0.63 \pm 0.043$ & $2.4 \pm 0.12 \pm 1.2 \pm 0.6$ & 0.3 & 0.038 \\
\hline$\chi_{b 0} \rightarrow \chi_{c 2} \chi_{c 2}$ & $0.0032_{-0.0012}^{+0.0038} \pm 0.035 \pm 0.0016$ & $0.13 \pm 0.033 \pm 0.066 \pm 0.033$ & 0.017 & $6.8 \times 10^{-4}$ \\
\hline$\chi_{b 1} \rightarrow h_{c} \psi$ & $0.18_{-0.0077}^{+0.0016} \pm 0.13 \pm 0.091$ & $0.88 \pm 0.078 \pm 0.17 \pm 0.22$ & 0.68 & - \\
\hline$\chi_{b 1} \rightarrow h_{c} \psi(2 S)$ & $0.089_{-0.0037}^{+0.00076} \pm 0.086 \pm 0.045$ & $0.67 \pm 0.18 \pm 0.13 \pm 0.17$ & 0.52 & - \\
\hline$\chi_{b 1} \rightarrow \eta_{c} \chi_{c 0}$ & $0.038_{-0.0055}^{+0.0048} \pm 0.038 \pm 0.019$ & $0.25 \pm 0.022 \pm 0.12 \pm 0.061$ & 0.19 & - \\
\hline$\chi_{b 1} \rightarrow \eta_{c}(2 S) \chi_{c 0}$ & $0.019_{-0.0027}^{+0.0023} \pm 0.022 \pm 0.0093$ & $0.17 \pm 0.046 \pm 0.12 \pm 0.043$ & 0.13 & - \\
\hline$\chi_{b 1} \rightarrow \eta_{c} \chi_{c 2}$ & $0.11_{-0.0066}^{+0.0031} \pm 0.075 \pm 0.055$ & $0.48 \pm 0.042 \pm 0.24 \pm 0.12$ & 0.37 & - \\
\hline
\end{tabular}


TABLE 1. (Continued)

\begin{tabular}{|c|c|c|c|c|}
\hline Reaction & $\Gamma_{\mathrm{NRQCD}}, \mathrm{eV}$ & $\Gamma_{\mathrm{LC}}, \mathrm{eV}$ & $\mathrm{Br}_{\mathrm{LC}}, 10^{-5}$ & $\operatorname{Br}_{\mathrm{LC}}(\psi \psi), 10^{-5}$ \\
\hline$\chi_{b 1} \rightarrow \eta_{c}(2 S) \chi_{c 2}$ & $0.054_{-0.0032}^{+0.0015} \pm 0.051 \pm 0.027$ & $0.33 \pm 0.089 \pm 0.23 \pm 0.083$ & 0.26 & - \\
\hline$\chi_{b 1} \rightarrow \chi_{c 0} \chi_{c 1}$ & $0.08_{-0.018}^{+0.022} \pm 0.061 \pm 0.04$ & $0.12 \pm 0.015 \pm 0.06 \pm 0.03$ & 0.091 & $4.2 \times 10^{-4}$ \\
\hline$\chi_{b 1} \rightarrow \chi_{c 1} \chi_{c 2}$ & $0.018_{-0.00087}^{+0.0015} \pm 0.028 \pm 0.0091$ & $0.23 \pm 0.03 \pm 0.11 \pm 0.057$ & 0.18 & 0.013 \\
\hline$\chi_{b 2} \rightarrow \eta_{c} \chi_{c 1}$ & $0.26_{-0.015}^{+0.0073} \pm 0.18 \pm 0.13$ & $0.63 \pm 0.011 \pm 0.31 \pm 0.16$ & 0.31 & - \\
\hline$\chi_{b 2} \rightarrow \eta_{c}(2 S) \chi_{c 1}$ & $0.13_{-0.0075}^{+0.0036} \pm 0.12 \pm 0.064$ & $0.35 \pm 0.044 \pm 0.24 \pm 0.086$ & 0.17 & - \\
\hline$\chi_{b 2} \rightarrow \chi_{c 0} \chi_{c 2}$ & $0.076_{-0.017}^{+0.02} \pm 0.058 \pm 0.038$ & $0.049 \pm 0.0075 \pm 0.025 \pm 0.012$ & 0.025 & $6.5 \times 10^{-5}$ \\
\hline$\chi_{b 2} \rightarrow \eta_{c} \eta_{c}$ & $0.26_{-0.069}^{+0.069} \pm 0.69 \pm 0.13$ & $0.64 \pm 0.02 \pm 0.31 \pm 0.16$ & 0.32 & - \\
\hline$\chi_{b 2} \rightarrow \eta_{c}(2 S) \eta_{c}$ & $0.26_{-0.068}^{+0.068} \pm 0.7 \pm 0.13$ & $0.71 \pm 0.092 \pm 0.48 \pm 0.18$ & 0.36 & - \\
\hline$\chi_{b 2} \rightarrow \eta_{c}(2 S) \eta_{c}(2 S)$ & $0.062_{-0.017}^{+0.016} \pm 0.18 \pm 0.031$ & $0.2 \pm 0.068 \pm 0.17 \pm 0.051$ & 0.1 & - \\
\hline$\chi_{b 2} \rightarrow \psi \psi$ & $9.7_{-0.73}^{+0.87} \pm 6.9 \pm 4.9$ & $9.6 \pm 0.42 \pm 0.33 \pm 2.4$ & 4.8 & 4.8 \\
\hline$\chi_{b 2} \rightarrow \psi(2 S) \psi$ & $9.6_{-0.72}^{+0.86} \pm 9.3 \pm 4.8$ & 11. $\pm 1.9 \pm 0.35 \pm 2.8$ & 5.7 & 3.3 \\
\hline$\chi_{b 2} \rightarrow \psi(2 S) \psi(2 S)$ & $2.3_{-0.17}^{+0.21} \pm 3.5 \pm 1.2$ & $3.4 \pm 1.4 \pm 0.094 \pm 0.84$ & 1.7 & 0.56 \\
\hline$\chi_{b 2} \rightarrow h_{c} h_{c}$ & $0.061_{-0.012}^{+0.012} \pm 0.17 \pm 0.031$ & $0.48 \pm 0.034 \pm 0.13 \pm 0.12$ & 0.24 & - \\
\hline$\chi_{b 2} \rightarrow \chi_{c 0} \chi_{c 0}$ & $0.0021_{-0.00044}^{+0.00037} \pm 0.0037 \pm 0.0011$ & $0.013 \pm 0.0019 \pm 0.0065 \pm 0.0032$ & 0.0063 & $1.1 \times 10^{-6}$ \\
\hline$\chi_{b 2} \rightarrow \chi_{c 1} \chi_{c 1}$ & $0.026_{-0.0074}^{+0.0069} \pm 0.063 \pm 0.013$ & $0.28 \pm 0.03 \pm 0.14 \pm 0.069$ & 0.14 & 0.018 \\
\hline$\chi_{b 2} \rightarrow \chi_{c 2} \chi_{c 2}$ & $0.028_{-0.0052}^{+0.0038} \pm 0.042 \pm 0.014$ & $0.54 \pm 0.11 \pm 0.27 \pm 0.13$ & 0.27 & 0.011 \\
\hline$\chi_{b 2} \rightarrow h_{c} \psi$ & $1.1_{-0.14}^{+0.12} \pm 1 . \pm 0.57$ & $3.6 \pm 0.09 \pm 0.68 \pm 0.9$ & 1.8 & - \\
\hline$\chi_{b 2} \rightarrow h_{c} \psi(2 S)$ & $0.56_{-0.069}^{+0.057} \pm 0.62 \pm 0.28$ & $2.1 \pm 0.36 \pm 0.39 \pm 0.52$ & 1. & - \\
\hline$\chi_{b 2} \rightarrow \chi_{c 1} \chi_{c 2}$ & $0.044_{-0.0015}^{+0.0008} \pm 0.036 \pm 0.022$ & $0.49 \pm 0.1 \pm 0.24 \pm 0.12$ & 0.25 & 0.018 \\
\hline
\end{tabular}

exclusive bottomonium decay is set up by the mass of the decaying bottomonium meson, but not by the double pole mass of $b$-quark $2 m_{b}$. (3) Within NRQCD both approaches are equivalent at the leading order approximation. In paper [1] we used an incorrect value of the $M=2 m_{b}$ to calculate the width of the considered decays within LC. To correct this error one should replace $m_{b}$ by a half of the mass of the decaying bottomonium in all expressions for the amplitudes and the widths.

It should be noted that with the corrections described above, our analytical results are in agreement with the analytical ones of papers [2-4]. The resulting numerical values of decay widths and branching fractions change significantly (see Table I). Now the corrected theoretical predictions are in reasonable agreement with experimental results [5]. Figure 2 in [1] should also be changed and rescaled by factor $\sim 2 \times 10^{-3}$.

[1] V. V. Braguta, A. K. Likhoded, and A. V. Luchinsky, Phys. Rev. D 80, 094008 (2009).

[2] J. Zhang, H. Dong, and F. Feng, Phys. Rev. D 84, 094031 (2011).

[3] W.-L. Sang, R. Rashidin, U-R. Kim, and J. Lee, Phys. Rev. D 84, 074026 (2011).

[4] L.-B. Chen and C.-F. Qiao, arXiv:1204.0215.

[5] C. P. Shen, C.Z. Yuan, and T. Iijima (Belle Collaboration), Phys. Rev. D 85, 071102(R) (2012). 